\begin{tabular}{lc}
\hline & Sharif University of Technology \\
SCIENTIA & Transactions B: Mechanical Engineering \\
IRAN ICA & www.scientiairanica.com \\
\hline
\end{tabular}

\title{
An alternative mechanism for the formation of high density lipoprotein in peripheral tissue
}

\author{
B. Damirchi ${ }^{a}$, M.S. Saidi ${ }^{a, *}$, M. Rismanian ${ }^{a}$, B. Firoozabadi ${ }^{a}$ and M. Amininasab $^{b}$ \\ a. School of Mechanical Engineering, Sharif University of Technology, Tehran, Iran. \\ b. Department of Cell and Molecular Biology, Faculty of Science, University of Tehran, Tehran, Iran.
}

Received 19 May 2014; received in revised form 15 February 2015; accepted 19 September 2015

\section{KEYWORDS}

High density

lipoprotein;

Structural modes;

Lipid protein complex;

Assembly.

\begin{abstract}
High Density Lipoprotein (HDL) is a lipid-protein complex responsible for transporting cholesterol and triglyceride molecules, as these compounds are unable to dissolve in aqueous environments such as a bloodstream. Among the most well-known possible structures, the belt-like structure is the most common shape proposed for this vital bimolecular complex. In this structure, the protein scaffold encompasses the lipid bilayer and a planar circular structure is formed. Several HDL simulations with embedded components in the lipid section were performed. Here, we applied a series of molecular dynamic simulations using the MARTINI coarse grain force field to investigate an HDL model, with pores of different radii in the bilayer section instead of embedded components. The results of such studies revealed the probable structural modes in HDL configurations. In addition, totally, $2.5 \mu \mathrm{s}$ simulations led to a study of the ratio of lipids to protein in HDL conformation, determination of the structural shape of HDL, and the stability of each model due to atomic interaction. Furthermore, we proposed a new conformation for HDL during its initial steps of construction outside the cells and in peripheral tissue.
\end{abstract}

(C) 2016 Sharif University of Technology. All rights reserved.

\section{Introduction}

High density lipoprotein, known as HDL, is a key component of blood, and plays a vital role in collecting cholesterol during circulation in the bloodstream and delivering it to the liver, where it is degraded or prepared for removal from the body [1]. Thus, it is usually named, "good cholesterol". This function of HDL reduces coronary disease and hardening of the arteries i.e. the existence of HDL is inversely related to the risk of its associated diseases [2]. Apolipoprotein A-I (APO A-I) protein, which is the main component of HDL, is synthesized inside the liver and intestine. Due to the efflux of lipids and cholesterol from peripheral tissue, the primary discoidal shape of HDL begins to

\footnotetext{
*. Corresponding author. Tel.: +98 2166165558 ;

Fax: +982166000021
}

E-mail address: mssaidi@sharif.edu (M.S. Saidi) form $[1,3]$. As this process continues and more lipids and cholesterol absorb and aggregate, HDL particles alter into a spherical shape [1]. APO A-I is comprised of 243 amino acid residues with a 43 residue globular Nterminal domain (which is not involved in lipid binding) and a 200 residue lipid binding C-terminal domain [4]. It is predicted that among these residues, 43 of them are not involved in lipid binding sites [5].

As it is difficult to observe the molecular structure of HDL, Molecular Dynamics (MD) approaches, which are based on computational methods and computer simulations, are suitable. Several models are introduced for HDL conformation, including the picketfence $[6,7]$, in which lipid acyl chains are parallel to the protein scaffold, helical-hairpin [8,9] and doublebelt structures, in which the lipids are wrapped by two belts of the protein scaffold [10].

The nanodisc double belt model, which is of interest in the current study, has a self-assembly 
process and its size is controlled by the scaffold protein [11]. Experiments and computational achievements suppose that $11-22$ out of 200 residues of the binding domain do not participate in binding to the lipid molecules [12]. Therefore, nanodiscs comprised of truncated scaffold protein are called $\operatorname{MSP} 1 \Delta(1-11)$ and $\operatorname{MSP} 1 \Delta(1-22)$, in which 11 and 22 residues from the C-terminal domain are truncated, respectively.

Molecular Dynamics (MD) simulations offer an operative approach to the visual dynamics of several biomolecules in specific processes [13]. All Atom (AA) simulations reflect the properties and structures of a desired biomolecule, such as HDL. However, this approach is limited to a few nanoseconds, and, for processes such as HDL self-assembly, longer simulation (hundreds of nanoseconds) is needed. Therefore, the Coarse-Grain (CG) approach is investigated to model and study the HDL structure. Shih et. al [4] performed $\mathrm{CG}$ simulations to consider the structure of $\mathrm{HDL}$ and to study the self-assembly phenomenon of various nanodiscs. Their study reveals that CG modeling of the HDL structure is in high agreement with experiments, and the idea worked well. Furthermore, they studied MSP1, MSP1 $\Delta(1-11)$ and MSP1 $\Delta(1-22)$ models of HDL and analyzed the stability of each. In MSP1, the rings overlap at the ends of the protein scaffold rings. However, in $\operatorname{MSP} 1 \Delta(1-22)$, there is a gap between the tails of each ring in the protein section. Moreover, there is neither gap nor overlap in the scaffold protein in MSP1 $\Delta(1-11)$. Hydrophilic and hydrophobic interactions are predominant factors in the assembly of a nanodisc. Jones et al. studied the temperature influence on the HDL structure and per-residue helix stability [14]. Koivuniemi et al. considered the concentration of core lipids in the surface monolayer and the interfacial tension of droplets resembling HDL particles [15]. Several properties of HDL are investigated, such as interfacial tension [16], cholesterol ester transport [17], cholesterol efflux roll on HDL configuration [18], and inter-ring rotation of the two alpha-helical monomers about the lipid unilamellar bilayer core [19]. In addition, the lipidprotein ratio in HDL has been considered in several pieces of research $[1,4]$. Formation of HDL particles due to lipid flux and their interaction with protein scaffold was investigated. It was shown that lipid aggregation overtime leads to the creation of micelles, and the aggregation of micelles cause the protein scaffold to reshape into a ring style, wrapping the lipids in the middle [4].

In the present investigation, a series of simulations is performed on a nanodisc double-belt model of HDL with various pores in the dipalmitoylphosphatidylcholine (DPPC) bilayer section. A complete complex of APO AI and DPPC, comprised of 160 DPPC particles, was studied by Bayburt et al. [20]. Furthermore, they investigated several areas of research showing that the full and stable HDL particle consists of 160 DPPC lipids. We used the MSP $1 \Delta(1-11)$ HDL model with pores in different radii. These pores can be made when HDL is considered a carrier, and the cargo (e.g. drug) is released from the molecule. It is shown that the pores disappear due to high aggregation speed in the lipid domain. As previously mentioned, the protein tails reached each other without any gap or overlap. Our interest is to consider whether the nanodisc will remain in its superficial shape or if it would bend or the belts separate and build new conformations. This study reveals probable structural modes in proteinlipid complexes, such as HDL.

\section{Materials and methods}

Since it is a formidable undertaking to study the detailed interaction or structure of biomolecules at the molecular level, Molecular Dynamics (MD) simulations are used. Corresponding force field parameters are based on experiments and calculated with high accuracy to be consistent with them. Although the AA modeling of a biological phenomenon and correlated simulation results lead to a more detailed and precise understanding, in some cases, computational costs confine the researchers to applying a more effective tool. Taking this problem into account, Coarse Grain (CG) approaches have attracted researchers. In processes where general properties with less detail result in a high order of time scales, the CG approach is an efficient method to apply. Several CG mappings are introduced. Marink et al. [21] proposed a semiquantitative representation for lipid simulations. In this modeling, amino acid residues are considered as 2 beads (one for the backbone and one for side chains). Recently, a more precise representation of residues is introduced, known as the MARTINI technique [22]. In this technique, 4 heavy atoms are considered as one bead and a mass of 72 amu and 4 water molecules are mapped into one bead. Amino acid residues are mapped into 2-5 beads (one for the backbone and 24 for side chains) reflecting the more accurate properties of each residue compared to former mappings. Although the MARTINI technique, as a coarse grain method, falls short in detail, it has done well in biological simulations. In the present work, MARTINI mapping is used to study lipid aggregation in truncated models of $\operatorname{MSP} 1 \Delta(1-11)$.

\subsection{Model}

Shih et al. provided the all-atom structure of a complete model (without pore). Later, they introduced a coarse grain model of HDL, applying the MARTINI mapping on the all atom structure [1]. The initial model was comprised of $160 \mathrm{DPPC}$ and scaffold protein 


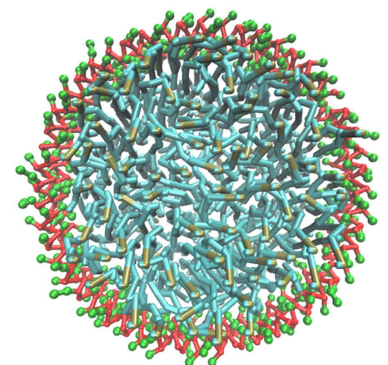

(a)

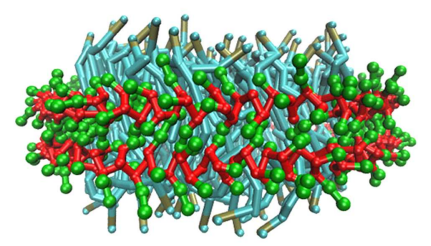

(b)
Figure 1. Complete MSP1 $\Delta(1-11)$ without pore. DPPC particles are shown in cyan and brown. Scaffold protein backbones are indicated in red and green depicts protein side chains: (a) Up view of $\operatorname{MSP} 1 \Delta(1-11)$ that illustrates the circular shape of the complete model; and (b) side view of MSP1 $\Delta(1-11)$ that shows the superficial shape of the complete model. Two scaffold protein rings wrapped the DPPC particles and bind them in hydrophobic section that causes the whole structure to be soluble in water and blood stream.

wrapping the lipid bilayer. Eleven residues of the lipid binding domain, as discussed previously, are truncated. Figure 1 shows the complete MSP1 $\Delta(1-11)$ (without pore). As depicted, two protein rings encompass the lipid bilayer section.

A complete initial model of $\operatorname{MSP} 1 \Delta(1-11)$ was used to generate our simulation models. A series of simulations were carried out on HDL, with eccentric pores of average radiuses $0,1.2,1.5,1.7$ and $2.0 \mathrm{~nm}$ in the lipid domain. 0, 14, 22, 28 and 42 DPPC particles are omitted from the complete $\operatorname{MSP} 1 \Delta(1-11)$, respectively. Models are constructed using the VMD program [23], and Table 1 depicts the simulation models. The results reveal probable structural modes and propose specific formations for varying lipid/protein ratios in lipid-protein complexes. In order to study the behavior and conformation of the models, each of them was simulated for 500 microseconds, which is discussed in the following sections.

\subsection{Simulation protocol}

Model parameters were obtained from the MARTINI forcefield [4]. Moreover, all simulations were performed using NAMD 2.9 [24]. The model consists of a hexagonal periodic water box containing apolipoprotein A-I protein, a DPPC lipid bilayer and solvent molecules. The $9.6 \mathrm{~nm}$ diameter APO A_I protein and DPPC lipid bilayer are solvated using TIP3p water molecules in a box of dimensions $131 \times 131 \times 83 \AA^{3}$. $15 \%$ of the water beads are anti-freeze CG beads. The proper amount of counter ions was added to satisfy the model's electroneutrality. In addition, structures contain between 10000 and 11000 CG particles after solvation and ionization. The water box was extended $\pm 15 \AA$ from each side of the protein, and a periodic boundary condition is exerted. A cutoff of $12 \AA$ was applied to calculate non-bonded interaction, with shifting beginning at $9 \AA$ to ensure a smooth cutoff. Simulations were performed using a $20 \mathrm{fs}$ time step. In addition, systems were minimized for 5000 time steps, taking into account that neither protein nor lipids were constrained. Langevin dynamics, with a damping coefficient of $1 \mathrm{ps}^{-1}$, was used to maintain a constant temperature of $323 \mathrm{~K}$. Also, a constant pressure of $1 \mathrm{~atm}$ was sustained with a Nose-Hoover Langevin piston with a period of $2000 \mathrm{zfs}$ and a decay time of 1000 fs. Each simulation lasts up to $500 \mathrm{~ns}$. Furthermore, it should be noted that all simulations were carried out in a NPT ensemble.

\section{Results}

Simulations on truncated nanodiscs with an eccentric pore of different radii were performed to study lipidprotein behavior and interaction, which led to a specific shape for each of them. In this study, different pores were created in the lipid section to investigate whether lipids aggregate together or tend to keep the previous conformation and build semi-micelle structures in the inner radius of truncated HDL; thus, the HDL structure remaining superficial and its nanodisc structure turning into a nanoring shape. As mentioned before, pores are made after releasing the cargo in peripheral tissue. However, lipids aggregate and cause the whole structure to bend, which leads to new conformations. It is worth mentioning that in all simulations, the pore filled due to lipid aggregation in less than $1 \mathrm{~ns}(0.2 \%$ of simulation time), which shows the high tendency of lipids to stay together. Furthermore, system conformational models are revealed and discussed. The results of such studies may also provide an insight

Table 1. Configuration of performed simulations.

\begin{tabular}{ccccc}
\hline $\begin{array}{c}\text { Simulation } \\
\text { name }\end{array}$ & $\begin{array}{c}\text { System } \\
\text { configuration }\end{array}$ & DPPC & $\begin{array}{c}\text { Truncated } \\
\text { radius }\end{array}$ & $\begin{array}{c}\text { Total simulation } \\
\text { time }\end{array}$ \\
\hline Sim A & $\operatorname{MSP} 1 \Delta(1-11)$ & 160 & $0 \AA$ & $500 \mathrm{~ns}$ \\
Sim B & $\operatorname{MSP} 1 \Delta(1-11)$ & 146 & $12 \AA$ & $500 \mathrm{~ns}$ \\
Sim C & $\operatorname{MSP} 1 \Delta(1-11)$ & 138 & $15 \AA$ & $500 \mathrm{~ns}$ \\
Sim D & $\operatorname{MSP} 1 \Delta(1-11)$ & 132 & $17 \AA$ & $500 \mathrm{~ns}$ \\
Sim E & $\operatorname{MSP} 1 \Delta(1-11)$ & 118 & $20 \AA$ & $500 \mathrm{~ns}$ \\
\hline
\end{tabular}




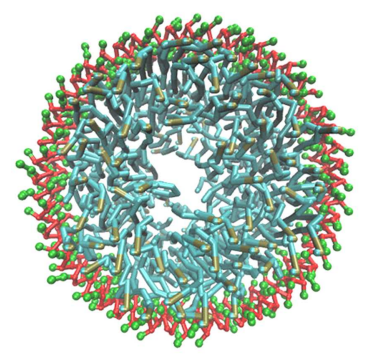

(a)

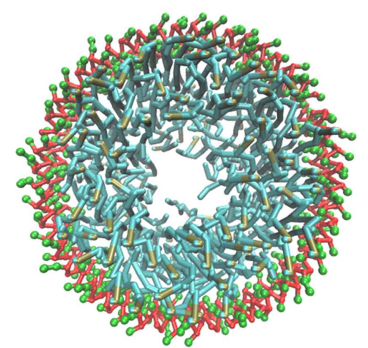

(d)

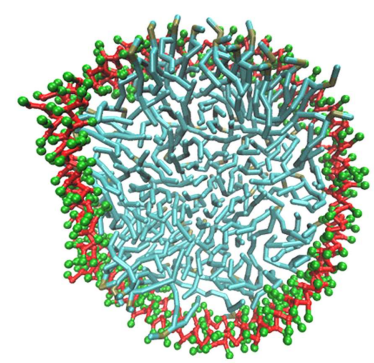

(b)

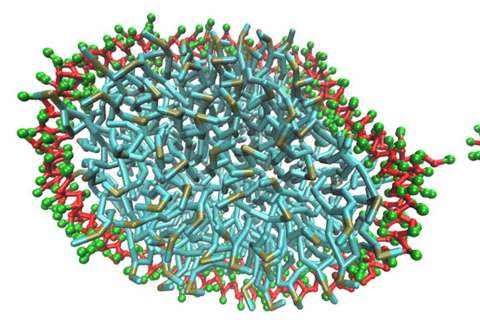

(e)

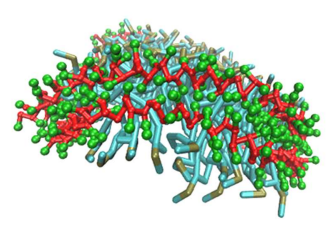

(c)

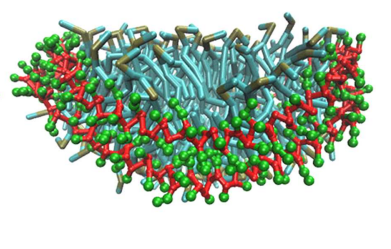

(f)

Figure 2. Initial and final shapes of Sim B and Sim C. First row illustrates the conformational changes of Sim B: (a) Up view of initial condition of HDL model with a pore average radius of $1.2 \mathrm{~nm}$; (b) up view of Sim B after $500 \mathrm{~ns}$ simulation. The pore is filled and circular shape of the initial state is almost kept; and (c) side view of Sim B after 500 ns simulation which shows lipid aggregation causes curvature due to hydrophobic and hydrophilic interactions. Second row depicts the structural changes of Sim C: (d) Up view of HDL model with a pore average radius of $1.5 \mathrm{~nm}$ in lipid section; (e) up view of Sim C after $500 \mathrm{~ns}$ that shows the pore is completely filled due to lipid aggregation; and (f) side view of Sim C after the simulated time. The structure warped more than the previous model to gain its equilibrium state.

into hydrophobic and hydrophilic interaction in lipidprotein complexes, such as HDL.

As previously studied by Shih et al., in Sim A, in which a complete HDL with no pore was simulated, the structure remains planar and in a nanodisc shape [1]. A full model of HDL (results not shown) is considered in order to have a check point in simulation parameters, leading to a planar shape for this model.

During $500 \mathrm{~ns}$ simulation, lipids in $\mathrm{Sim} \mathrm{B}$, in which 14 DPPC particles are omitted and 146 DPPC particles remain, aggregate immediately. Figure 2(a)(c) illustrate the initial and final shape of Sim A after $500 \mathrm{~ns}$ simulations. As shown, the lipids aggregate and fill the pore. Furthermore, the whole structure slightly warps to countervail the omitted lipid spaces. It can be implied that even though some HDL particles are deleted from the bilayer section, the lipids expand and the planar shape is almost kept.

Figure 2(d)-(f) demonstrate the evolution in Sim $\mathrm{C}$ from the initial condition to its final formation. In this model, there are 138 DPPC particles in the middle. Similar to Sim B results, lipid aggregation fills the pore in less than a nanosecond. Additionally, the lack of sufficient lipids to construct a fully planar shape leads the structure to warp more than previous simulations, due to more deleted lipids. Furthermore, it causes contraction, which will be discussed later. The results show that for HDL, the deleted lipids do not reach a critical amount, due to which, the system begins to bend and tends to warp the structure to keep its circular shape. Therefore, warping is one of the probable modes of the complex. In order to consider a more equilibrated and realistic condition, for HDL with 146 DPPC particles, another run setup is investigated. In this set up, the scaffold protein was restrained and the whole structure was simulated under a NVT ensemble for $10 \mathrm{~ns}$. Then, a $490 \mathrm{~ns}$ simulation was carried out in the NPT ensemble without restraint. The results were almost identical for both simulations due to the precipitate aggregation of DPPC particles (less than $1 \mathrm{~ns}$ ). The same aggregation pace can be seen for other models, which means that such an existing pore is far from reality, in most cases, but that there would be negligible error due to the immediate aggregation of lipids.

Figure 3(a)-(c) show the Sim D conformations for varying periods of time. After a few nanoseconds, the pore is filled by lipid aggregation. Then, the structure begins to contract due to hydrophilic and hydrophobic interaction. This interaction leads the model to bend and the lipids from each side of the bent HDL to interact with each other. Therefore, significant distortions cause the initial discoidal shape to alter to a semi-micelle conformation. This simulation reveals that a critical amount of lipids in the HDL configuration has been reached, wherein the structure should bend and that warping alone does not satisfy the equilibrium state. Finally, after 500 ns simulations 


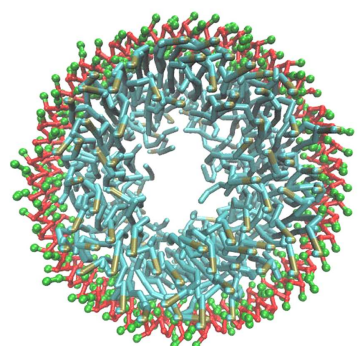

(a)

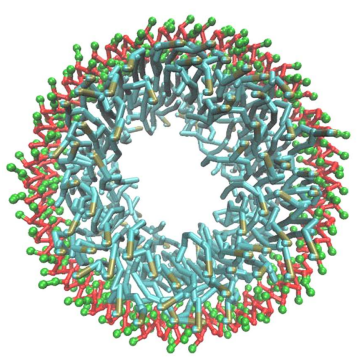

(d)

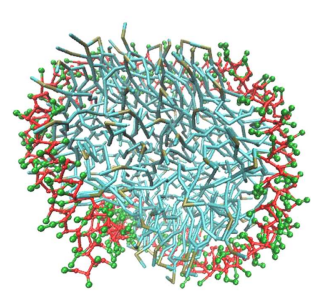

(b)

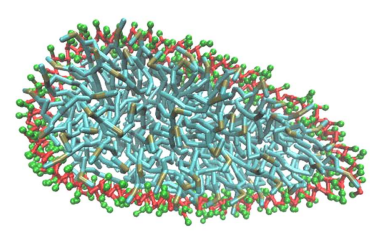

(e)

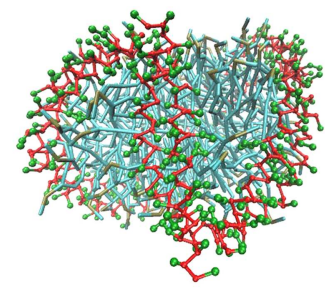

(c)

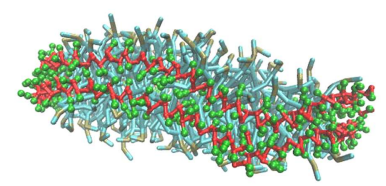

(f)

Figure 3. Sim D and Sim B, initial and final formations: (a) Initial up view; (b) final up view; and (c) final side view of HDL model with average pore radius of $1.7 \mathrm{~nm}$ in DPPC section. The results of this simulation reveal the mode of lipid-protein complex in which the lipid aggregation leads to a semi-micelle structure encompassed by protein scaffold: (d) Initial up view; (e) final up view; and (f) final up view of HDL model with a pore of average radius of $2.0 \mathrm{~nm}$ in lipid section after $500 \mathrm{~ns}$ simulation. The results indicate another structural mode of lipid-protein complex in which the superficial shape is almost kept and circular formation altered to an elliptic conformation.

of Sim D, the lipids are contracted and the scaffold protein encompasses the lipids in a flexuous manner. Comparing the configurations in several frames, this semi-micelle structure seems to be the final shape of Sim D. This result shows one mode of the protein-lipid complex that may occur.

Structural changes of Sim E are illustrated in Figure 3(d)-(f). In this model, about 42 lipids are omitted. The snapshots reveal that after the pore is filled by lipid aggregation, the structure tends to bend to reach its equilibrium state. However, in continuation, the dominant mode alters to a new conformation, which is a superficial elliptical structure. Despite other modes in previous simulations, this structure neither warps to keep its circular shape nor bends due to lipid aggregation. This is also another probable mode of configuration for a truncated HDL to reach its equilibrium state.

\section{Discussion}

In the previous section, results of several simulations were described for truncated HDL with various initial conformations. As mentioned previously, several modes of lipid-protein complex were revealed by CG simulation. The simulations also indicate that not only do 160 DPPC lipids in a lipid-protein complex such as HDL lead to a nanodisc and planar structure, but that, also, there are other composition ratios which lead to superficial shapes, not necessarily in a circular form.
Elliptic structures of HDL configurations may occur before completion of HDL at the outer layer of cells and peripheral tissue, where initial steps to constructing a nanodisc structure are in progress. On the other hand, when lipid efflux from peripheral tissue aggregate to construct larger colonies in contact with HDL protein, it can be suggested that, at first, a superficial elliptical structure may be formed. Then, as more lipids join the structure, nanodisc structures which have a circular and planar shape are created. Now, we consider some properties of the simulations and discuss them in detail to determine what is exactly happening at a molecular scale.

\section{Root Mean Square Deviation (RMSD)}

RMSD is calculated to consider whether they have reached an equilibrium state. Figure 4 illustrates RMSD for all structures for lipid and protein scaffolds. As shown, the RMSD for structures with a pore do not reach an asymptotic value, and there are still fluctuations and steeps. This means that the simulated time is not sufficient to reach an equilibrium state for the models. Shih et al. [12] also proposed that the required time for HDL structures to reach an equilibrium state is about 10 microseconds using the diffusion theory. The calculated RMSD, relative to bisecting the plane, revealed that the models reach their equilibrium state within 100 ns. Figure 4(a) shows that as the pore radii increases, the required time for the whole structure equilibrium state rises consequently 


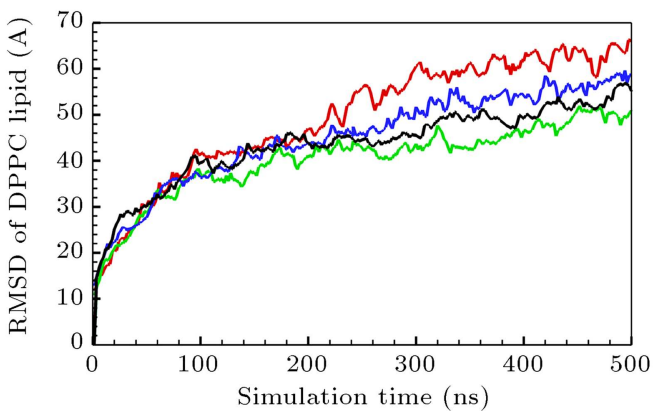

(a)

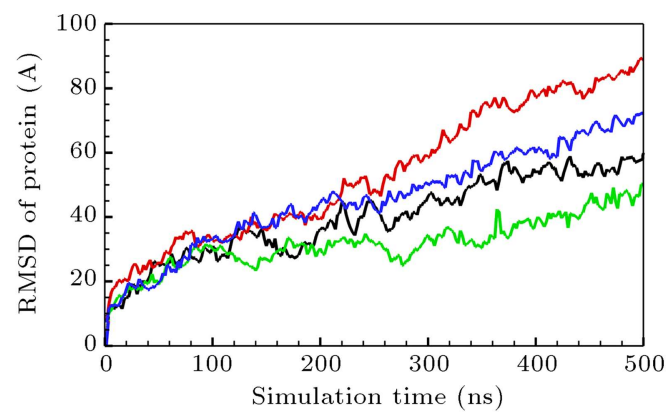

(b)

Figure 4. Root mean square deviation of HDL models with pores of various radiuses. HDL structure with pore radius of $1.2 \mathrm{~nm}$ is depicted in green, HDL model with pore radius of $1.5 \mathrm{~nm}$ is indicated in black, HDL model with pore radius of $1.7 \mathrm{~nm}$ is shown in red, and HDL model with pore radius of $2.0 \mathrm{~nm}$ is illustrated in blue: (a) RMSD values for DPPC particles (lipids). It is obvious that the RMSD of lipids do not reach an asymptotic value due to insufficient simulation time; and (b) RMSD values of protein scaffolds for pore accommodated models. The least RMSD value belongs to Sim B with the least pore radius and the highest value is correlated to Sim D where semi-micelle final configuration is obtained.

for DPPC particles. This is due to the needed time for lipid aggregation to fill the pore and balance the interactions, particularly hydrophobic and hydrophilic interactions. Figure 4(b) depicts the correlated RMSD diagram for scaffold protein. The initial fact that can be concluded from the diagram is that, as the pore radius increases, the protein further deforms, due to interaction and lipid aggregation. Since the protein rings encompass the lipids, the Sim B model obtains the least RMSD values, due to less deleted lipids, as it has the closest formation to the complete model (160 DPPC). The increase in deleted DPPC particles leads to more deformations and, consequently, as the protein scaffold wraps the lipids, it faces deformation. The exception is for Sim D, where we have a model with an average pore radius of $1.7 \mathrm{~nm}$. As shown in the previous section, the final shape of the simulated model is a semi-micelle lipid configuration, and the protein scaffold wraps the lipids in a flexuous style. In this simulation, the most deformation and distortion occurred due to lipid aggregation. Taking into account the final formation of this simulation, it is obvious why the RMSD values of the protein scaffold of Sim D have the highest value amongst all others.

\section{Interaction energy}

Interaction energy is calculated for each model, which is comprised of VDW and the electrostatic interaction of the DPPC particles, with respect to other lipids and protein scaffolds. Figure 5 demonstrates the calculated interaction energy per lipid values during the simulation. For models with 146 and 138 lipids (Sim B and Sim C, respectively) the minimum energy values are obtained. Between these 2 models, the HDL model, with a pore average radius of $1.2 \mathrm{~nm}$, has the minimum interaction energy.

We conclude that as the number of lipids decreases, generally, an equilibrium state is formed in

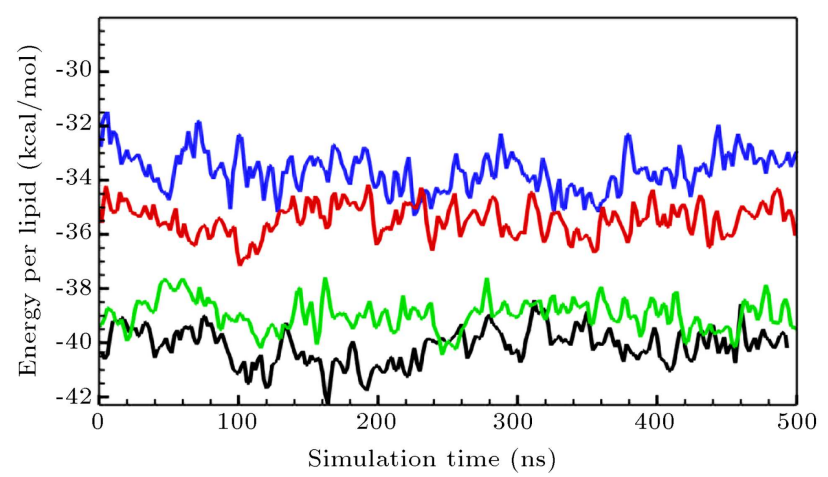

Figure 5. Interaction energy per lipid during simulations in models with pore. HDL model with a pore of average radius of $2.0,1.7,1.5$ and 1.2 are depicted as blue, red green and black, respectively. As the number of deleted lipids increases, the interaction energy per lipids rises.

higher interaction energies. This means that as the structure starts to form a complete and equilibrated formation (160 DPPC), interaction energies decline, which can be interpreted as a stronger attraction between the particles. This fact is starkly in agreement with equilibrium simulation results in which systems are inclined to reach lower energies, and corresponding structures are the dominant formation in nature. For the HDL model with 132 DPPC lipids, which faces significant structural changes, the equilibrium state is approachable at higher potential. Besides, for the last model, higher potential energy is needed to reach a superficial elliptical structure.

\section{Radius of gyration}

In order to consider the compactness of the protein scaffold, the radii of gyration are calculated for Sim $\mathrm{B}$ and Sim C models during 500 ns simulation time. These two structures have the most similar shapes, and the calculations were investigated to study whether the scaffold protein compacts to compensate for the 


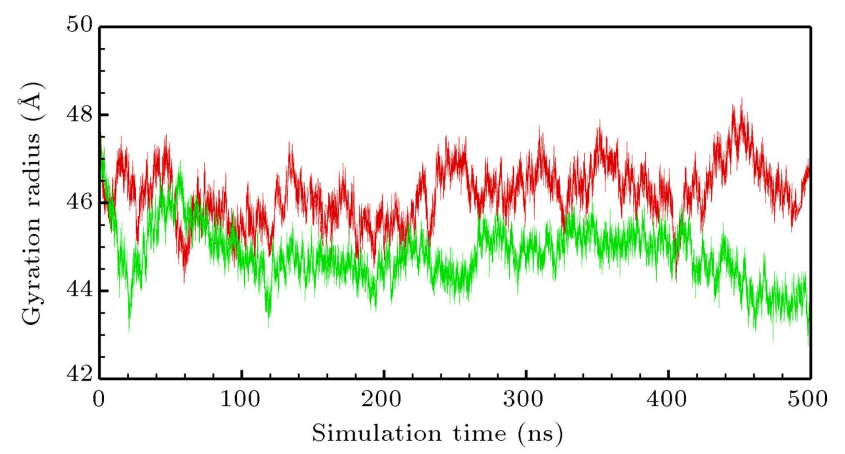

Figure 6. Radii of gyration for HDL models with 146 and 138 DPPC particles in lipid domain. Sim B model is shown in red and Sim $\mathrm{C}$ model is depicted in green colors.

omitted lipids or whether warping alone is the dominant process leading to an equilibrium state. Figure 6 demonstrates the radii of gyration for the discussed models.

According to Figure 6, the radius of gyration decreases as the number of omitted DPPC particles rises. It can be concluded that by compacting the scaffold protein, in addition to warping the structure, both occur in order to reach an equilibrium state. So, there is another parameter to investigate the overall volume changes of the structure, which will be discussed in the following section.

\section{Density}

Number density for both Sim B and Sim C are calculated to consider volume changes. Figure 7 illustrates the number of density of DPPC particles along the $x$ coordinates for both models.

As indicated in the figure, compared to Sim C, Sim B lipid number density has higher values. Thus, it can be concluded that even though scaffold protein contracts and the average radius of HDL reduces, the density of the DPPC particles does not remain con-

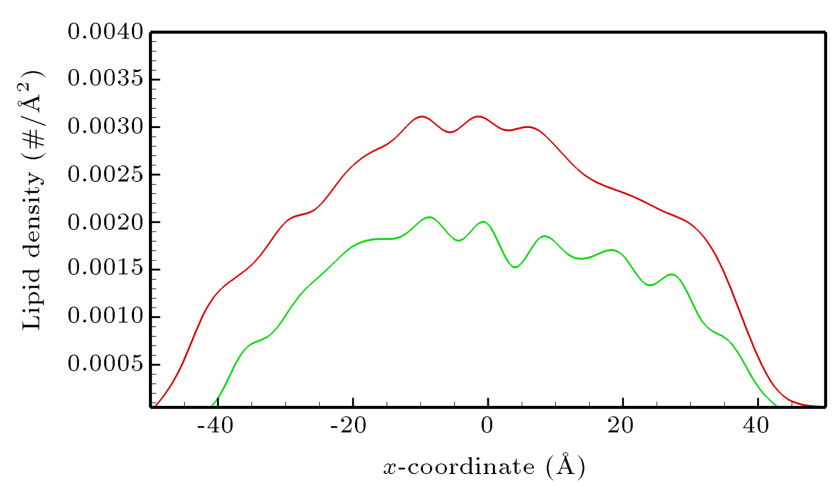

Figure 7. Lipids density along $x$-coordinate for Sim B and Sim C simulations. Sim B and Sim C simulation models are represented in red and green, respectively. This can be concluded that reducing density to keep semi-planar shape is an alternative way to reach the equilibrium state. stant. In summary, constriction of the whole structure, as well as local expansion in the lipid section (albeit in opposite directions), leads to final conformation of the HDL.

\section{Conclusion}

We have investigated a coarse grain molecular dynamics simulation on nanodisc formation of HDL with various pores in the lipid section. The MARTINI force field proved that it is a suitable technique to study biomolecules, such as proteins and lipids. Several coarse grain simulations were carried out to study the protein-lipid ratio in the structural shape of high density lipoprotein, known as HDL. Simulations reveal that there are several conformational modes in this vital biomolecule. We proposed that, first, a planar elliptic shape is formed outside the cells, and, as more lipids are absorbed by the structure, a circular formation begins to form, respectively. For this particular model, which is a MSP1 HDL model with 11 residues truncated in the lipid binding site, 160 DPPC particles are needed to construct a fully nanodisc structure. In addition, it is shown that as the complex goes forward to form a circular formation, lipid density varies to satisfy the equilibrium state in such a way that for almost equivalent areas, density decreases, compared to higher lipid-protein ratios. The simulated time for each model ( $500 \mathrm{~ns})$ was not sufficient to reach the equilibrium state; nevertheless, the structures gained their stable formation during the simulation due to similar conformations in periods of time. Such simulations could reveal a probable way of how HDL forms in a peripheral environment and illustrates the dominant role of hydrophilic and hydrophobic interaction in lipid-protein complexes.

\section{References}

1. Shih, A.Y., Freddolino, P.L., Arkhipov, A. and Schulten, K. "Assembly of lipoprotein particles revealed by coarse-grained molecular dynamics simulations", J. of Struc. Biology, 157(3), pp. 579-592 (2007).

2. Linsel-Nitschke, P. and Tall, A.R. "HDL as a target in the treatment of atherosclerotic cardiovascular disease", Nature Reviews Drug Discovery, 4(3), pp. 193205 (2005).

3. Davidson, W.S. and Silva, R.G.D. "Apolipoprotein structural organization in high density lipoproteins: Belts, bundles, hinges and hairpins", Current Opinion in Lipidology, 16(3), pp. 295-300 (2005).

4. Shih, A.Y., Arkhipov, A., Freddolino, P.L. and Schulten, K. "Coarse grained protein-lipid model with application to lipoprotein particles", J. of Phys. Chem. $B, \mathbf{1 1 0}(8)$, pp. 3674-3684 (2006). 
5. Segrest, J.P. et al. "The amphipathic $\alpha$ helix: A multifunctional structural motif in plasma apolipoproteins", Advances in Protein Chemistry, 45, pp. 303-369 (1994).

6. Segrest, J.P. "Amphipathic helixes and plasma lipoproteins: Thermodynamic and geometric considerations", Chem. and Phys. of Lipids, 18(1), pp. 7-22 (1977).

7. Phillips, J.C., Wriggers, W., Li, A. and Schulten, K. "Predicting the structure of apolipoprotein AI in reconstituted high-density lipoprotein disks", Biophys. Journal, 73(5), pp. 2337-2346 (1997).

8. Brouillette, C.G., Anantharamaiah, G.M., Engler, J.A. and Borhani, D.W. "Structural models of human apolipoprotein AI: A critical analysis and review", Biochimica et Biophysica Acta (BBA)-Molecular and Cell Biology of Lipids, 1531(1), pp. 4-46 (2001).

9. Rogers, D.P., Roberts, L.M., Lebowitz, J., Engler, J.A. and Brouillette, C.G. "Structural analysis of apolipoprotein AI: Effects of amino- and carboxyterminal deletions on the lipid-free structure", $J$. of Biochem., 37(3), pp. 945-955 (1998).

10. Segrest, J.P., Jones, M.K., Klon, A.E., Sheldahl, C.J., Hellinger, M., Loof, H. and Harvey, S. "A detailed molecular belt model for apolipoprotein AI in discoidal high density lipoprotein", J. of Biological Chem., 274(45), pp. 31755-31758 (1999).

11. Denisov, I.G., Grinkova Y.V., Lazarides, A.A. and Sligar, S.G. "Directed self-assembly of monodisperse phospholipid bilayer Nanodiscs with controlled size", $J$. of the American Chemical Society, 126(11), pp. 3477-3487 (2004).

12. Shih, A.Y., Denisov, I.G., Phillips, J.C., Sligar, S.G. and Schulten, K. "Molecular dynamics simulations of discoidal bilayers assembled from truncated human lipoproteins", Biophysical J., 88(1), pp. 548-556 (2005).

13. Th $\phi$ gersen, L., Schi $\phi$ tt, B., Vosegaard, T., Nielsen, N.C. and Tajkhorshid, E. "Peptide aggregation and pore formation in a lipid bilayer: A combined coarsegrained and all atom molecular dynamics study", Biophysical J., 95(9), pp. 4337-4347 (2008).

14. Jones, M.K., Catte, A., Patterson, J.C., Gu, F., Chen, J., Li, L. and Segrest, J.P. "Thermal stability of apolipoprotein AI in high-density lipoproteins by molecular dynamics", Biophysical J., 96(2), pp. 354371 (2009).

15. Koivuniemi, A., Sysi-Aho, M., Oresic, M. and Olila, S. "Interfacial properties of high-density lipoprotein-like lipid droplets with different lipid and apolipoprotein AI compositions", Biophysical J., 104(10), pp. 21932201 (2013).

16. Ollila, O., Lamberg, A., Lehtivaara, M., Koivuniemi, A. and Vattulainen, L. "Interfacial tension and surface pressure of high density lipoprotein, low density lipoprotein, and related lipid droplets", Biophysical J., 103(6), pp. 1236-1244 (2012).
17. Koivuniemi, A., Heikelä, M., Kovanen, P.T., Vattulainen, I. and Hyvönen, M.T. "Atomistic simulations of phosphatidylcholines and cholesteryl esters in highdensity lipoprotein-sized lipid droplet and trilayer: Clues to cholesteryl ester transport and storage", Biophysical J., 96(10), pp. 4099-4108 (2009).

18. Cuellar, L.Á., Prieto, E.D., Cabaleiro, L.V. and Garda, H.A. "Apolipoprotein AI configuration and cell cholesterol efflux activity of discoidal lipoproteins depend on the reconstitution process", Biochimica et Biophysica Acta (BBA) - Molecular and Cell Biology of Lipids, 1841(1), pp. 180-189 (2014).

19. Caulfield, T.R. "Inter-ring rotation of apolipoprotein AI protein monomers for the double-belt model using biased molecular dynamics", J. of Molecular Graphics and Modelling, 29(8), pp. 1006-1014 (2011).

20. Bayburt, T.H., Grinkova, Y.V. and Sligar, S.G. "Selfassembly of discoidal phospholipid bilayer nanoparticles with membrane scaffold proteins", Nano Letters, 2(8), pp. 853-856 (2002).

21. Marrink, S.J., Vries, A.H. and Mark, A.E. "Coarse grained model for semiquantitative lipid simulations", J. of Physical Chem. B, 108(2), pp. 750-760 (2004).

22. Marrink, S.J., Risselada, H.J., Yefimov, S., Tieleman, D.P. and Vries, A.H. "The MARTINI force field: Coarse grained model for biomolecular simulations", $J$. of Physical Chem. B, 111(27), pp. 7812-7824 (2007).

23. Humphrey, W., Dalke, A. and Schulten, K. "VMD: Visual molecular dynamics", J. of Molecular Graphics, 14(1), pp. 33-38 (1996).

24. Phillips, J.C., Braun, R., Wang, W., Gumbart, J., Tajkhorshid, E., Villa, E., Chipot, C., Skeel, R.D., Kale, L. and Schulten, K. "Scalable molecular dynamics with NAMD", J. of Computational Chem., 26(16), pp. 1781-1802 (2005).

\section{Biographies}

Behzad Damirchi received his BS degree in Mechanical Engineering from Khaje-Nasir University of Technology, Tehran, Iran, in 2009, and his MS degree from the Center of Excellence in Energy Conversion in the School of Mechanical Engineering at Sharif University of Technology, Tehran, Iran, in 2013. His main research interest is molecular biophysics.

Mohammad Said Saidi is Professor of Mechanical Engineering at Sharif University of Technology, Tehran, Iran. His research interests include modeling and numerical analysis of transport and deposition of aerosol particles, and modeling and numerical analysis of biofluids.

Milad Rismanian received his BS degree in Mechanical Engineering from Bu-Ali Sina University, Hamadan, Iran, in 2009, and his MS degree from the Center 
of Excellence in Energy Conversion in the School of Mechanical Engineering at Sharif University of Technology, Tehran, Iran, in 2013. His research interests are molecular modeling, particle transport, nano-micro mechanics and biological heat transfer.

Bahar Firoozabadi is Professor of Mechanical Engineering at Sharif University of Technology, Tehran, Iran. Her research interests include: modeling and numerical analysis of biofluids, particle laden flow and density current in settling tanks.

Mehria Amininasab is Assistant Professor of Cell and Molecular Biology at Tehran University, Tehran, Iran. His research interests are biophysics, protein structures, protein-protein interaction, protein dynamics, enzymes, protein chemistry, channels, venoms, proteomics, and amyloids. 